\title{
Dark Matter from Our Probabilistic Gravity
}

\author{
Shantilal Goradia \\ Gravity Research Institute, Inc., Mishawaka, IN 46545-8102, USA
}

\begin{abstract}
Existence of dark stuff (dark matter + dark energy) is overwhelming. The WIMPs (Weakly interacting massive particles) hypnotized for the existence of dark stuff have not been detected. We are exploring the linkage of our quantum mechanical probabilistic and gravity to the issue of dark stuff in the universe. Our new horizon gives us a deeper insight, pointing to small black holes as candidates of dark stuff. We invoke the spooky nature of quantum physics which gets spookier to hold the universe together.
\end{abstract}

Key words: Quantum information, relativity and gravitation, dark matter, PACS numbers: 03.67.-a, 95.30.Sf, 95.35.+d.

\section{Introduction}

The author (described as "we" all along) derived the strong coupling $\left(C_{s}\right)$ by a quantum mechanical modification of Newtonian gravity [1]. We extend our derivation towards the issue of dark matter. We refer to an interesting quotation: "The really hard problems are great,” Mike Turner said, “Because we know they'll require a crazy new idea" [2]. The author of the book with that quotation (Mr. Panek) adds: "What science needed now wasn't the next Einstein but the next Newton to codify the math of this new universe" [2]. Here, we put both of these encouragements in perceptive, the latter one by taking a multifaceted Newtonian approach.

"There is on-going research for the detection of WIMPs based on the speculative idea of supersymmetry, which attempts to unify the fundamental forces of Nature, including gravity. The detection of WIMPs is expected to find a solution to the issue of dark stuff. We continue to hold and support our view of the millennium that gravity is not a fundamental force of Nature. We are therefore exploring baryons as the particles to address the issue of dark matter [3]. We struggle to point out baryons (nucleons) and end up in qualifying hypothetical small black holes as the candidates of dark matter.

Corresponding author: Shantilal Goradia, president, research field: theoretical physics.

\section{Dark Matter from Our Probabilistic Gravity}

Newton, wondered how "action at a distance", creates gravity without any visible action. Particles of matter must be extending and interacting for these actions to happen. Newtonian gravity is equivalent to particles (mainly nucleons), extending to interact and create forces like $C_{s} \mathrm{~s}$ [1]. The abundance of dark stuff implicitly suggests that we need to account for about 20 times more $C_{s}$ s, making us wonder how " 20 actions at a distance" take place without seeing any of these actions. That is the dilemma caused by the observation of dark stuff. Our theory stating: "The probability of interaction between two nucleons is inversely proportional to the square of their separation in Planck lengths" [1], which can be restated by adding the part in bold to address the dark stuff: "The probability of interactions between two nucleons is inversely proportional to the square of their separation in Planck lengths, with no limit on the sum of interactions that one nucleon undergoes with other nucleons in the universe." If all nucleons in the universe were laid next to each other in a line, Euler's series expansion for the sum of infinite inverse squares would give us the limit of the sum of probabilities of first nucleon to interact with all other nucleons in the universe simultaneously as 1.645. The problem is that nucleons are not laid that way. Therefore, instead of going from theory to predict 
observation, we have to go from observation to theory. Since the observed abundance of the dark stuff is 20 times the luminous matter, each nucleon must be interacting with 20 nucleons simultaneously. A simplifying example: Each nucleon must be like a superman with 20 hands; not a common man using one hand as implicitly assumed for pulling other nucleons. This would also explain how quantum particles hold the universe together. Bonds between 20 different cosmological entities in the universe can be attributed to these multiple interactions by one nucleon. A simplifying example of the bondage: If 20 hypothetical family members of each person were spread in different arbitrary locations in the world, the world would be better bonded.

\section{Points of Nucleon Interactions}

Symmetry dictates that nucleon extensions from a luminous nucleon would be radially divergent, but not extending in perfect straight lines due to mutual attractions along the way, making them curve somewhat like the way shown in Fig. 1 (part of the default background image from an HP Computer). Tiny bulb like points at their ends represents their hypothetical points of interactions where they would generate $C_{s}$ s. Since gravity is dark, we never know exactly where they interact.

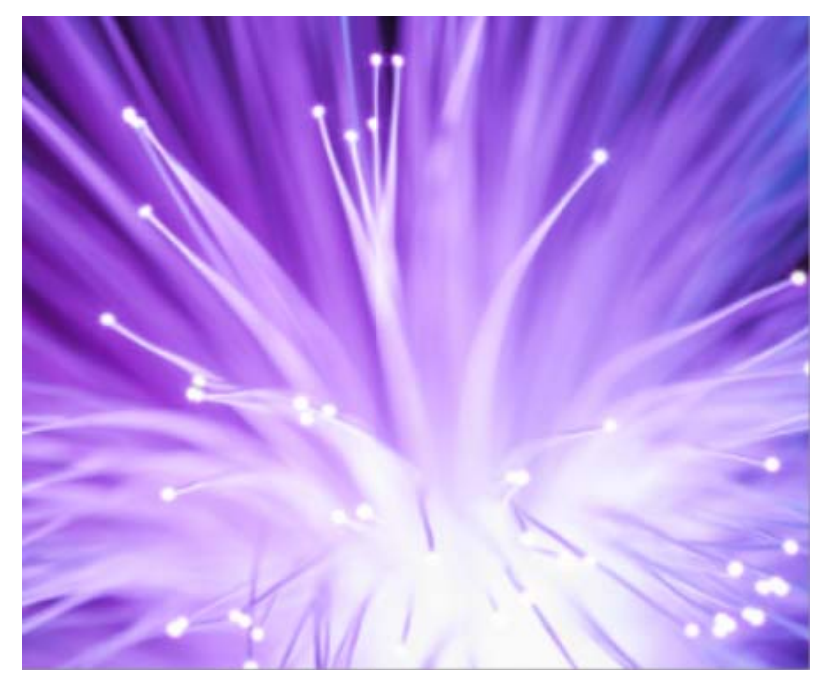

Fig. 1 Mental image of spooky nucleons extending from luminous matter.
If the nucleon extensions were radially divergent straight lines, most of them would miss to interact as they would keep on separating more and more with time. Their curvature in space would make some interact near the star of their origin; others (1) in the galaxies, (2) beyond the galaxies, and (3) potentially, beyond the preexistent universe. Unlike the cosmological constant, the number 20 (or whatever its exact equivalent) may not be a constant in space and time; that is another issue.

\section{Interactions Create Black Holes}

We find that the tiny bulb like points are small black holes. Here is why. "A black hole is formed when an aggregate of mass $M$ is confined within a radius of

$$
R=2 \mathrm{MG} / \mathrm{c}^{2}
$$

where, $G$ is the gravitational constant and $c$ is the speed of light [4]. The nucleons interact via their tiny quantum mouths [1]; therefore we consider that the size of their mouths $(R)$ is one Planck length i.e. $R=$ $10^{-35}$ meter. The aggregate of $M$ (mass of nucleon = $\left.1.67 \times 10^{-27} \mathrm{~kg}\right)$ with $G=\left(6.675 \times 10^{-11} \mathrm{~m}^{3} \cdot \mathrm{kg}^{-1} \cdot \mathrm{s}^{-2}\right)$ and $c=\left(300 \times 10^{6}(\mathrm{~m} / \mathrm{s})^{2}\right)$ is $2 \mathrm{MG} / \mathrm{c}^{2}=2.48 \times 10^{-22} \mathrm{~m}$. Since $10^{-35} \mathrm{~m}$ is obviously less than $2.48 \times 10^{-22} \mathrm{~m}$, the aggregate of mass $M$ is confined in one Planck length, making the quantum mouth a "small black hole", a candidate of the dark stuff. We cite one reference to support its candidacy: "The nature of dark matter remains unknown. Many ideas have been proposed including various types of baryonic and non-baryonic dark matter. Examples of low-luminosity discrete objects include planets, brown dwarfs, low-mass stars, neutron stars and small black holes” [5]. Therefore, the tiny bulb like points are small black holes, the candidates of dark stuff. The value of $C_{s}$ is $10^{40} \mathrm{~g} \cdot 10^{40}$ $\mathrm{g}$ is a high intensity force comparable to a black hole. It may appear and disappear every Planck time, one hundred million, trillion, trillion, trillionth $\left(10^{44} \mathrm{nth}\right)$ of a second, at far away points of nucleon interactions. Its long range effect is so small that it is mistaken strictly as a short range phenomenon. $10^{40} \mathrm{~g}$ diminishes to $1 \mathrm{~g}$ 
(Newtonian gravitation) at 1,000 femtometers (the radius of atoms) [6]. Regardless, $C_{s} \mathrm{~s}$ are black holes as explained, and are, therefore, the candidates of dark stuff. $20 \times 10^{79}$ small black holes resulting from all nucleon interactions in the universe are invisible (dark) to us except they create gravitational effects throughout the universe.

\section{Aspects of Gravitation}

\subsection{Information Paradox}

Considering gravitational lensing is ubiquitous to dark matter, what Einstein's genius figured out decades ahead of time of the discovery of dark matter was the effect of dark matter. The famous quote to explain general relativity in one sentence approved by Einstein is: "space tells mass how to move and mass tells space how to curve" which is equivalent to "dark matter tells luminous matter how to move and luminous matter tells dark matter how to curve the path for it to move.”The word "telling" implies "sending information". Information must prevail everywhere. We derived fine structure constant as $1 / 137.046$, close to its real value 1/137.04 [7]. We could not do that without first applying quantum informatics to cosmology. We are implicitly incorporating information here. The ON and OFF blinking of the tiny bulb like points in Fig. 1 is analogous to the OPEN and CLOSE positions of the quantum mouths in [7]. Talking about information, Dr. Hooft, a Nobel Laureate comments -"Most physicists agree that a black hole should be described by a Schrödinger equation, with a Herminean Hamiltonian, but this requires a modification of general relativity. Both General Relativity and Quantum Mechanics are shaking on their foundations [8]." He repeated his view this August in Stockholm to the best of our knowledge. We are not modifying general relativity. We are making quantum physics spookier, while spooky it already is.

\subsection{Classical Formulations of Gravity}

Newtonian gravity works well in the solar system where the mass of the system is concentrated at the central point, while General Relativity does a superb job explaining the perihelion of Mercury in addition to solar gravitation. MOND (Modification of Newtonian dynamics) does well in the galaxies [9]. The mass distribution is along the galactic disc. Why different formulations work differently at different scales? Fig. 1 implies that the tendency of nucleons extending to remote places and interacting there may not be mathematically consistent with any given classical formulation of gravity for all distances from one Planck length on. "The amount of dark matter in the universe determines the overall curvature of space. This is because dark matter produces gravity and gravity curves space" [10]. We say gravity links to $C_{S} s$ $[1,6,7,11]$. The common word "gravity" in both sentences relates strong couplings $\left(C_{s} \mathrm{~s}\right)$ to dark stuff; not as $1: 1$ but as $1: 20$, considering the (19 times more) abundance of dark stuff, enabling our probabilistic gravity to explain the dark stuff in its entirety without the need of a hypothetical particle.

\section{The Source of Our Information}

While trying to save the draft file of this article this October, a window popped up saying that the source of this information is invalid; there is no evidence, so the file will not be saved. We printed the file. The source of this article is a series of our new ideas that cannot be listed as a long list of references. Questionable old ideas resist justifiable new ideas from coming to light. Illustration: lack of evidence of the roundness of earth, before Columbus discovered the shape of the earth, could have delayed the ideas of gravitation.

\section{Conclusions}

The candidates for dark stuff are small black holes created by nucleon interactions; and the abundance of dark matter makes quantum mechanics spookier than previously thought, further explaining its capability to hold the universe together. 


\section{References}

[1] Goradia, S. 2012. "Newtonian Gravity in Natural Units.” Journal of Physical Science and Application 2 (7): 265-8.

[2] Panek, R. 2011. "The 4\% Universe, Dark Matter, Dark Energy, and the Race to Discover the Rest of Reality.” Mariner Books, Houghton Mifflin Harcourt, New York, 242.

[3] Goradia, S. 2016. American PhysicalSociety, Log number: MAR16-2015-004448 (abstract submitted).

[4] Harwit, M. 1998. "Astrophysical Concepts.” Springler-Verlag New York, Inc., New York, 41.

[5] Longair, M. 2006. "The Cosmic Century - A History of Astrophysics and Cosmology" Cambridge University Press, Cambridge CB2 8RU, UK, 250.

[6] Goradia, S. 2001. "Microscopic Implications of General Relativity.” In Proceedings of the Eighth international Conference on Particles, Strings and Cosmology,
University of North Carolina, Chapel Hills, 355-8.

[7] Goradia, S. 2015. "Decoding the Information of Life." Journal of Physical Science and Application 5 (3): 191-5.

[8] Hooft, G. T. 2006. "Black Holes and the Information Paradox." In Proceedings of the Sixth International Symposium, "Frontiers of Fundamental and Computational Physics, B. M. Birla Science Center, India, 29.

[9] Keeton, C. 2014. "A Ray of Light in a Sea of Dark Matter.”Rutgers University Press, New Brunswick, NJ, 7.

[10] Tucker, W. and Tucker K. "The Dark Matter, Contemporary Science's Quest for the Mass Hidden in Our Universe.” William Morrow and Company, Inc., New York, 171.

[11] Goradia, S. 2000. "Consistent Equation of Classical Gravitation to Quantum Limit and Beyond.” In Proceedings of the Fourth International Symposium on Frontiers of Fundamental Physics, Hyderabad, India, 345-8. 